\title{
Monoclonal Antibody Directed against a Membranous Protein of MARC-145 Cells Blocks Infection by PRRSV
}

\author{
DOMINIC THERRIEN AND SERGE DEA
}

Centre de Recherche en Microbiologie et Biotechnologie, INRS-Institut Armand-Frappier, Université du Québec, Laval, Québec, H7V $1 B 7$

\section{INTRODUCTION}

In its natural host, infection by the porcine reproductive and respiratory syndrome virus (PRRSV) is restricted to cells of the macrophage/monocyte lineage and testicular germ cells (Plagemann 1996; Sur et al., 1996). In vitro, only cell subclones derived from the established monkey kidney epithelial cell line MA-104, such as MARC-145 and CL2621, and primary cultures of porcine alveolar macrophages (PAMs) efficiently replicated the porcine arterivirus (Bautista et al., 1993). Factors implicated in this restricted tropism have not yet been elucidated, but PRRSV enters in PAMs and in MARC-145 cells through a mechanism of receptor-mediated endocytosis (Kreutz and Ackermann, 1996; Nauwynck et al., 1999). Recently, a $210 \mathrm{kDa}$ protein has been proposed as a putative receptor for PRRSV on PAMs, since a MAb directed against this protein blocked virus infection of PAMs (Duan et al., 1998). However, this MAb failed to react with MARC-145 and porcine monocytes, suggesting that PRRSV may enter in these cells by interacting with other cellular proteins. MAbs to Gp4 and Gp5 enveloped glycoproteins

of PRRSV neutralize viral infectivity, but the viral structural protein responsible for viral attachment is still to be identified (Meulenberg et al., 1997; Pirzadeh and Dea, 1997). In this study, the capacity of PRRSV to attach and infect different cell lines was first determined. Subsequently, two MAbs directed against a 60 to $66 \mathrm{kDa}$ membranous protein of MARC-145

The Nidoviruses (Coronaviruses and Arteriviruses).

Edited by Ehud Lavi et al. Kluwer Academic/Plenum Publishers, 2001. 
cells were found to interfere with viral infection, thus representing a putative receptor or co-receptor for PRRSV.

\section{METHODOLOGY AND RESULTS}

Virus binding to the host cells is a crucial step in the infection process. To study cell susceptibility to PRRSV, a binding assay was previously described to follow attachment of PRRSV on the surface of different cell lines by flow cytometry (Therrien et al., 2000). Infection was also performed on these cell lines to verify their capacity to replicate PRRSV. The cells were infected at a MOI of 0,01 with the Quebec strain, IAF-Klop. After $48 \mathrm{~h}$, cells were fixed with acetone to perform immunofluorescence (IIF) with anti-N MAb IAF-K8 or harvested to verify the presence of PRRSV genome by RT-PCR using oligonucleotide primers 1010 PLS and 1011PLR, designed to amplify a 434 bp DNA fragment encompassing the entire ORF7 gene and a portion of the 3' terminal non-coding region of PRRSV (Mardassi et al., 1994). Only MARC-145 cells, PAMs, and a small population of porcine peripheral blood leukocytes which has been previously characterised as monocytes (Therrien et al., 2000) were infected efficiently by PRRSV. However, other cell lines tested (PT, PK-15, BHK-21, 293A, and RK-13), apparently resistant to PRRSV infection, were also able to bind PRRSV on their surface.

To further investigate on the nature of the restricted tropism, MAbs were raised against cell surface proteins. Three injections of 50:g of cell lysates of MARC-145 and PAMs cells, or PRRSV semi-purified virus, were given to $\mathrm{Balb} / \mathrm{C}$ mice at 2 week-intervals, prior fusion experiments of spleen cells with Sp2/O myeloma cells. Two hybridomas, 18-A7 and 18-F6, were obtained that secreted MAbs to epitopes on the surface proteins of MARC-145 cells, but not on the surface of PAMs. Both MAbs blocked efficiently PRRSV infection of MARC-145 cells. The capacity of PRRSV to bind to MARC-145 cells was reduced by about $60 \%$ following preincubation of MAb 18-F6 (Fig1.D). Western blotting analyses were performed to identified membranous protein reacting with MAb 18-A7. MARC-145 cells were harvested with $20 \mathrm{mM}$ of EGTA pH8, $30 \mathrm{~min}$ at $37^{\circ} \mathrm{C}$, then solubilized with LB-2 buffer ( $20 \mathrm{mM}$ Tris- $\mathrm{HCl}, \mathrm{pH} 7.5,150 \mathrm{mM} \mathrm{NaCl}, 5 \mathrm{mM}$ EDTA, $0.5 \%$ NP-40, $0.1 \%$ Na-deoxycholate, $0.1 \%$ SDS) (Mardassi et al. 1996). The proteins were separated by electrophoresis on SDS-PAGE gel and transferred on nitrocellulose membrane. A protein with a size ranging from 60 to $66 \mathrm{kDa}$ was obtained. 

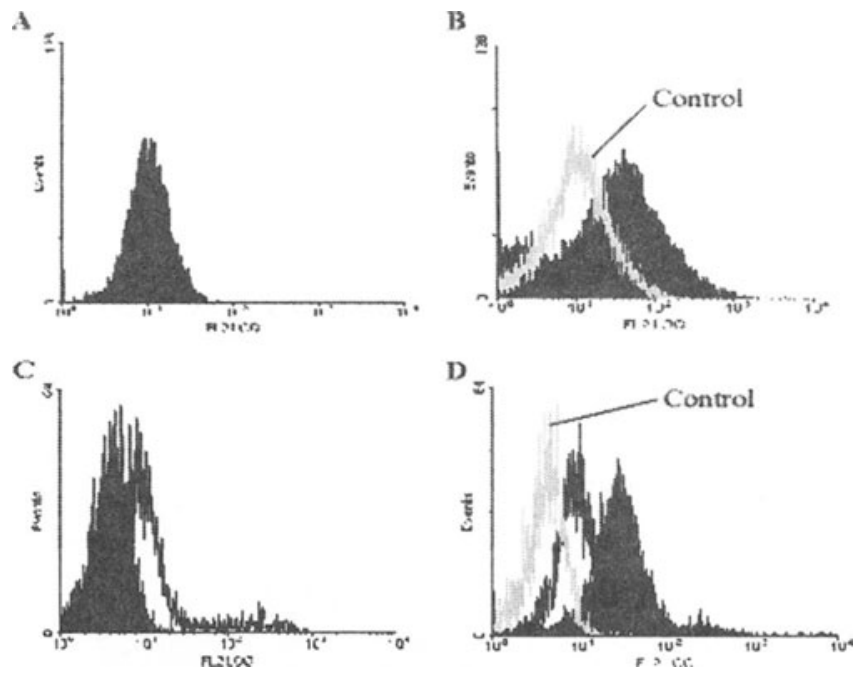

Figure 1. Binding of MAb 18-F6 and inhibition of PRRSV attachment. Suspensions of PAMs (A and B) or MARC-145 cells (C and D) were inoculated with the IAF-Klop strain of PRRSV at a MOI of $10 \mathrm{TCID}_{50}$ per cell. Following an adsorption period of $1 \mathrm{~h}$ at $4^{\circ} \mathrm{C}$, infected cells were washed with PBS and incubated with biotinylated MAb IAF-K8 for $30 \mathrm{~min}$ at $37^{\circ}$. The surface bound immune complexes were labelled with streptavidin-phycoerythrin, and fluorescent level was analyzed by flow cytometry. Filled patterns represent control (A and C) cells incubated in the presence of biotinylated MAb IAF-K8 and streptavidin-phycoerythrin. $\mathrm{B}$ and D. Cells were incubated with the Mab 18-F6 for $30 \mathrm{~min}$ at room temperature prior to the binding assay (black line). In this case filled patterns represent cells incubated with the virus.

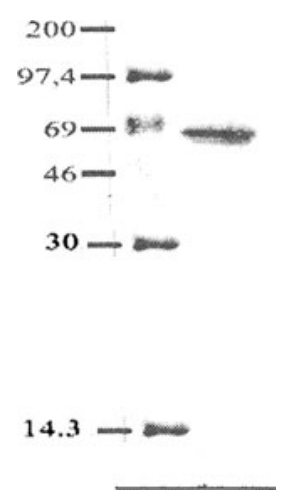

Figure 2. Reactivity of Mab 18-F6 against a cellular protein of 60 to $66 \mathrm{kDa}$. Western blot analysis of MARC-145 solubilized with LB-2 buffer $(20 \mathrm{mM}$ Tris- $\mathrm{HCl}, \mathrm{pH} 7.5,150 \mathrm{mM}$ $\mathrm{NaCl}, 5 \mathrm{mM}$ EDTA, $0.5 \% \mathrm{NP}-40,0.1 \%$ sodium deoxycholate, $0.1 \%$ SDS) (Mardassi et al. 1996). Following electrophoresis in $12 \%$ SDS-PAGE, proteins were electrophoretically trans-fered on nitrocellulose membrane, saturated with PBS containing $5 \%$ of fat milk and incubated with Mab 18-F6. The reactions was revealed following an incubation in the presence of horsedish peroxidase labelled caprine $\operatorname{IgG}$ directed against mouse $\operatorname{IgG}$, and staining of the immune complexes was obtained by a final incubation of the nitrocellulose membrane in a chloronaphtol solution containing $3 \% \mathrm{H}_{2} \mathrm{O}_{2}$. 


\section{DISCUSSION}

Interaction of viral particles to specific receptors on the surface of permissive cells is the primary step of a viral infection. In this study, it was shown that the capacity of a cell line to bind PRRSV was not sufficient to allow a productive infection. In fact, it has been demonstrated that some cell lines known to be resistant to PRRSV infection can also bind the virus. Transfection of PRRSV genome into BHK-21 or Vero cells, thus bypassing surface cellular receptors, results in a productive infection (Kreutz, 1998), suggesting that PRRSV infection is probably restricted by its capacity to attach or penetrate the cytoplasmic membrane of the host cell. Data obtained raise the hypothesis that more than one receptor may be involved in PRRSV infection. The MAb, 18-F6, directed against a protein of 60 to $66 \mathrm{kDa}$ on the surface of MARC-145 cells, was shown to block PRRSV infection. Since the MAb blocked only partially PRRSV binding, it could not be determined whether this protein is the initial binding factor or a co-receptor involved after binding of the virus. MAb 18-F6 failed to recognize an epitope on the surface of PAMs. Since a protein of $210 \mathrm{kDa}$ has been proposed to be a potential receptor for PRRSV on the surface of PAMs, results of this study indicate that PRRSV enters in the MARC-145 cells through the interaction with a different receptor. EAV and SHFV, but not LDV, have previously demonstrated their ability to infect MA-104 cells, from which MARC-145 cells have been established (Plagemann 1996). This raises the hypothesis that PRRSV, EAV and SHFV may enter by the same pathway in these epithelial cells via a common receptor. This common receptor could be the protein recognized by the MAb 18-F6, but this remains to be further investigated.

\section{ACKNOWLEDGMENTS}

This work was supported by the National Science and Engineering Research Council of Canada, strategic grant \#STP0202083. D. Therrien is a recipient of a $\mathrm{PhD}$ grant from the FCAR, Québec.

\section{REFERENCES}

Bautista, E.M., Goyal, S.M., Yoon, I.J, Joo, H.S., Collins, J.E. (1993) Comparison of porcine alveolar macrophages and CL2621 for the detection of porcine reproductive and respiratory syndrome (PRRS) virus and anti-PRRS antibody. J Vet Diagn Invest 5, 163-165. 
Duan, X., Nauwynck, H.J., Favoreel, H.W., Pensaert, M.B. (1998) Identification of a putative receptor for porcine reproductive and respiratory syndrome virus on porcine alveolar macrophages. $J$ Virol 72, 4520-4523.

Kreutz, L.C., Ackermann, M.R. (1996) Porcine reproductive and respiratory syndrome virus enters cells through a low pH-dependent endocytic pathway. Virus Res 42, 137-147.

Kreutz, L.C. (1998) Cellular membrane factors are the major determinants of porcine reproductive and respiratory syndrome virus tropism. Virus Res 53, 121-128

Mardassi H, Wilson L, Mounir S, Dea S (1994) Detection of porcine reproductive and respiratory syndrome virus and efficient differentiation between Canadian and European strains by reverse transcription and PCR amplification. J Clin Microbiol 32, 2197-2203,

Mardassi, H., Massie, B., Dea, S. (1996) Intracellular synthesis, processing and transport of proteins encoded by ORFs 5 to 7 of porcine reproductive and respiratory syndrome virus Virology 221, 98-112,

Meulenberg, J.J.M., Van Nieuwstadt, A.P., Van Essen-Zandbergen ,A., Langeveld, J.P.M. (1997) Posttranslational processing and identification of a neutralization domain of the GP4 protein encoded by ORF4 of Lelystad virus. J Virol 71, 6061-6067,

Nauwynck, H.J., Duan, X., Favoreel, H.W., Van Oostveldt, P., Pensaert, M.B. (1999) Entry of porcine reproductive and respiratory syndrome virus into porcine alveolar macrophages via receptor-mediated endocytosis. J Gen Virol 80, 297-305.

Pirzadeh, B., Dea, S. (1997) Monoclonal antibodies to the ORF5 product of porcine reproductive and respiratory syndrome virus define linear neutralizing determinants. $J$ Gen Virol 78, 1867-1873,

Plagemann, P.G.W. (1996) Lactate dehydrogenase-elevating virus and related viruses. Fields Virology $3^{\text {rd }}$ ed., Raven publishers, Philadelphia, pp. 1105-1120.

Sur, J.H., Doster, A.R., Christian, J.S., Galeota,J.A., et al. (1997) Porcine reproductive and respiratory syndrome virus replicates in testicular germ cells, alters spermatogenesis, and induces germ cell death by apoptosis. $J$ Virol 71, 9170-9179.

Therrien, D., St-Pierre, Y., Dea, S. (2000) Preliminary characterization of protein binding factor for porcine reproductive and respiratory syndrome virus on the surface of permissive and non-permissive cells. Arch. Virol. (in press) 